\title{
Latest Developments in Manufacturing and Recycling of Refractory Materials
}

\author{
CHAI REN (i) $^{1,3}$ and RAVI K. ENNETI ${ }^{2}$ \\ 1.-Xiamen Tungsten Co. Ltd, Chengdu, China. 2.-Global Tungsten and \\ Powders, Towanda, PA, USA. 3.—e-mail: chai.ren.cn@gmail.com
}

Renowned for their unique properties, refractory metals and materials have been widely used in electronic, nuclear, and defense industries. Manufacturing components from refractory metals presents great challenges due to their high melting temperature and low ductility. Until now, powder metallurgy followed by thermomechanical process has been the only viable route for producing bulk refractory materials on an industrial scale. However, efforts to develop new techniques or improve conventional processes are ongoing. Recently, due to advancements in fabrication equipment, many novel processing technologies such as additive manufacturing have been developed. Also, additives and alloying elements that either decrease manufacturing difficulties or improve material performance have proven to be valuable for refractory material systems.

This special topic on refractory metals and materials focuses on recent advances in overcoming process challenges or improving material performance. The papers are mostly devoted to manufacturing refractory materials in an innovative way or enhancing their properties by introducing additives or alloying elements. The collection presents ten papers covering the research areas of extraction, cermets, composites, high-entropy alloys, additive manufacturing, single-crystal materials, and refractory metal hydrides.

As a result of their combination of high hardness, good corrosion resistance, and excellent high-temperature performance, TiC cermets are important refractory materials for cutting tools and hightemperature applications. Addition of transitionmetal carbides and controlling the binder content

Chai Ren and Ravi Enneti are Guest Editors for the Refractory Metals and Materials Committee of TMS and organized the topic Latest Developments in Manufacturing and Recycling of Refractory Materials in this issue.

(Received August 30, 2021; accepted August 31, 2021; published online September 21, 2021) are conventional ways to improve the mechanical properties of TiC. In "Effect of Co and Ni Contents on the Sintering Behavior, Microstructure Evolution, and Mechanical Properties of (Ti,M)C-Based Cermets," Chen et al. performed a systematic study on the effect of binder $(\mathrm{Co}, \mathrm{Ni})$ content on the transverse rupture strength and fracture toughness of $\mathrm{TiC}$ with $\mathrm{WC}, \mathrm{Mo}_{2} \mathrm{C}$, and $\mathrm{VC}$ additions. They reported a correlation between the mechanical properties and microstructure of this (Ti,M)C-base cermet. In "Effect of In Situ Precipitated White Particles on Mechanical Properties of (Ti, V, W, Mo)C-based cermets," Chen et al. examined the effect of precipitated particles on the microstructure and mechanical properties of ( $\mathrm{Ti}, \mathrm{V}, \mathrm{W}, \mathrm{Mo}$ )C-based cermets, and demonstrated an improvement in strength, hardness, and fracture toughness as the Mo and WC content was increased up to $10 \mathrm{wt} . \%$

Compared with conventional elements, extraction of metals such as zirconium, niobium, and titanium leads to greater challenges. Due to their refractory nature, specially designed leaching and extraction processes using more aggressive conditions as well as flux media are required. In "Intensive Studies for Modeling and Thermodynamic of Fusion Digestion Processes of Abu Rusheid Mylonite Rocks," Eliwa et al. report an experimental and modeling study of the fusion digestion process of mylonite rocks from the Abu Rushied area of Egypt. The effectiveness of two types of flux alkali, viz. $\mathrm{NaOH}$ and $\mathrm{KOH}$, was compared, and the process parameters of time, temperature, alkali ratio, and mesh size optimized.

High-entropy alloys have been extensive studied recently. Solid-solution alloys containing four or more elements at near equiatomic ratio provide these systems with unique behaviors, including but not limited to mechanical properties, and corrosion or oxidation resistance. Among them, high-entropy alloys with refractory metal elements are of especial interest as they also inherit the high-temperature performance and other advantages from the refractories. In "Single-Phase Medium to High-Entropy 
Alloys Developed by a Simple Thermodynamic Approach," Moghaddam et al. worked on several VCrMo-based medium- to high-entropy alloys with both simulation and synthesis tools and propose a novel approach to design high-entropy alloys based on a binary system with addition of other principal elements based on thermodynamic parameters.

With its high melting temperature and ductile-tobrittle transition temperature (DBTT), additive manufacturing of tungsten was found to be challenging because the formation of defects such as cracks is nearly unavoidable. In addition, the poor mechanical properties of pure tungsten further limited the use of these three-dimensional (3D)-printed parts for any structural applications. In "Selective Laser Melting of Tungsten-Rhenium Alloys," Eckley et al. developed a new method to manufacture W-Re alloy parts directly using tungsten and rhenium powders, by selective laser melting and postprocessing heat treatments. The W-25\%Re alloy samples prepared using this method are reported to have near full density, smaller grain sizes, reduced crack number, and better mechanical behaviors than samples of pure tungsten or alloys with lower Re content.

Molybdenum's high melting point, good elevatedtemperature mechanical behavior, low thermal expansion coefficient, and large thermal conductivity make it an excellent candidate for high-temperature applications. The addition of a small amount of rareearth oxide can significantly improve its high-temperature performance and reliability. However, further increasing the oxide content in oxide-dispersion strengthened (ODS)-Mo material has proved to be difficult. In "Fabrication and Mechanical Properties

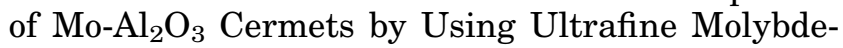
num and Nanosized Alumina Powders," Chen et al. reported a fabrication route combining planetary ball milling, carbothermic reduction, and sintering to prepare $\mathrm{Mo}-(\mathrm{Ni})-\mathrm{Al}_{2} \mathrm{O}_{3}$ material. This new type of Mo-based cermet has oxide content up to $30 \mathrm{wt}$.\%, along with hardness of 753.25 HV, which is over four times higher than pure molybdenum samples.

For refractory metals such as molybdenum and tungsten, thermomechanical processing is one of the very limited approaches to effectively improve the ductility. Therefore, the microstructure development during plastic deformation and annealing is of essential importance to truly understand the mechanism of ductilization for molybdenum and tungsten. In "The Recovery and Recrystallization Behavior of Cold-Compressed Mo-3Nb Single Crystal," Jiao et al. performed an electron backscattered diffraction (EBSD) and transmission electron microscopy (TEM) study on Mo-3Nb single crystals in their deformed and annealed states. The formation of grain boundaries during deformation and the evolution of substructures during annealing are discussed. The effect of compressive strain on recovery and recrystallization was analyzed systematically.
Zirconium hydride is known as an eligible moderator for thermal neutron reactors as a result of its high-temperature stability along with a unique large negative temperature coefficient of reactivity. It is generally synthesized by directly hydriding zirconium metal and power metallurgy techniques. However, the mechanical reliability of zirconium hydride parts prepared using this method suffers due to the cracks and defects formed during the hydriding process. In "Estimation of Fracture Toughness for $\varepsilon$ Zirconium Hydride by Vickers Microhardness Indentation Method," Cui et al. studied the Young's modulus and hardness of $\varepsilon$ zirconium hydride using the Olivier-Pharr theory and nanoindentation technique, and compared the results with conventional methods. The correlation between zirconium hydride microstructure and its crack propagation behavior is also discussed.

Mo-Si-B alloy is a unique type of refractory material. While it shares the desirable properties of high-temperature performance and oxidation resistance with conventional refractories, Mo-Si-B features a relatively low density, which makes it a more desired choice for use in many weight-sensitive fields. At the same time, its poor fracture toughness limits its potential for structural applications. In "Effect of $\mathrm{Ti}$ and $\mathrm{Si}$ Additions on Microstructure, Fracture Toughness, and Oxidation Resistance of Nb-Mo5SiB2 Composite," Lin et al. investigated the effect of Ti and Si addition on the toughness, oxidation resistance, and strength of $\mathrm{Nb}$ Mo5SiB2 composite. Based on the phase composition and fracture morphology, the strengthening and toughening mechanisms of the additives are also discussed systematically.

Reaction sintering of alumina and zircon resulted in a novel type of composite alumina-mullite-zirconia (AMZ), with a combination of high strength, good high-temperature performance, and excellent corrosion resistance. However, its relatively poor sintering behavior resulted in high porosity, thus limiting its applications. In "Introducing $\mathrm{MnO}_{2}$ and $\mathrm{ZnO}$ Additives for the Development of Alumina-Mullite-Zirconia Composites," Majidian et al. report a group of AMZ samples with addition of $\mathrm{MnO}_{2}$ or $\mathrm{ZnO}$. These additives improve the mechanical properties and thermal shock resistance of the composites through a complicated set of mechanisms, including formation of solid solution, grain size refinement, and mullite phase modification.

All the titles and authors of the articles published under this topic on the "Latest Developments in Manufacturing and Recycling of Refractory Materials" in the November 2021 issue (vol. 73, no. 11) of $J O M$ can be accessed fully via the journal's page at: http://link.springer.com/journal/11837/73/11/page/1.

Publisher's Note Springer Nature remains neutral with regard to jurisdictional claims in published maps and institutional affiliations. 